\title{
Immunotherapy-related adverse effects: how to identify and treat them in the emergency department
}

\author{
David H Henry, MD, ${ }^{a}$ interviews Maura Sammon, MD $^{\mathrm{b}}$
}

\begin{abstract}
${ }^{a}$ Department of Medicine, University of Pennsylvania Perelman School of Medicine; and ${ }^{\mathrm{b} L e w i s ~ K a t z ~ S c h o o l ~ o f ~ M e d i c i n e, ~ T e m p l e ~}$ University, both in Philadelphia, Pennsylvania
\end{abstract}

\begin{abstract}
When patients with cancer present to the emergency department with therapy-related symptoms, it's crucial to ascertain at the outset whether the treatment is with chemotherapy or immunotherapy so that the appropriate symptom treatment can be initiated as early as possible. In this interview, Dr David Henry and Dr Maura Sammon discuss some of the most common immunotherapyrelated side effects - lung, gastrointestinal, rash, and endocrine-related problems - and Dr Sammon describes in detail how physicians in the ED would triage and treat the patient. However, the overarching takeaway is the importance of communication: first, between the oncologist and patient, so that the patient is aware of these nuances in advance of an emergency, and second, between the ED physician and the treating oncologist soon after the patient has presented and undergone an initial assessment.
\end{abstract}

Dr Henry is the Editor-in-Chief of The Journal of Community and Supportive Oncology (www.jcso-online.com).This is an edited version of the interview podcast. You can listen to the audio of the original interview here.

DR HENRY I am pleased to be talking with Dr Maura Sammon, an emergency department (ED) physician, about identifying and treating immunotherapy-related side effects in the ED. This is a hot topic in oncology, and I was very interested in having an ED physician talk about what happens when treating oncologists send their patients to the ED, because a physician may think it is chemotherapy when it is immunotherapy. Let's start with the example of an oncology patient going to the ED with some symptoms, and the ED physician asks the patient what they're being treated with. The patient may or may not say the right thing - that is, inform you whether they are being treated with chemotherapy or immunotherapy. How do you morph over into knowing that they are not getting chemotherapy?

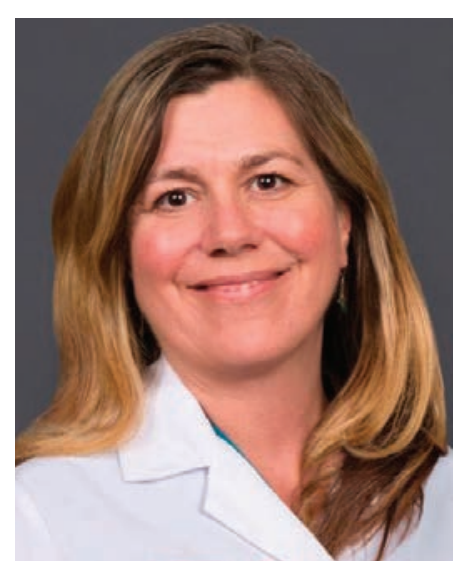

Dr Maura Sammon apy, when they're actually on immunotherapy, and it can really send the treatment team down the wrong path. I have a metaphor to explain this. They say that Great Britain and the United States are two nations separated by a common language. For example, when a British person talks about football, they mean something very different than when an American talks about football. If someone in Great Britain asks you to come play football, you might show up with shoulder pads and a helmet rather than shin guards, and you're left without having the right tools to participate in the game.

How this sometimes plays out with immunotherapy, unfortunately, is that a patient will present to the ED and say they're having a cough and that they're on chemotherapy for melanoma. Usually, this patient would be worked up for being in a potentially immunosuppressed state. You might get a white blood cell count. You might get a chest X-ray. You might see what looks to you like a new infiltrate

Correspondence: David H Henry, MD; David.Henry@uphs.upenn.edu. Disclosures: Dr Sammon and Dr Henry report no disclosures or conflicts of interest. JCSO 2018;16(4):e216-e220. (02018 Frontline Medical Communications. doi: https:// doi.org/10.12788/jcso.0408 
on this chest X-ray and then start going down the path of treating someone whom you think is immunosuppressed with pneumonia and giving them antibiotics rather than what could be life-saving steroids, as would be the case if the patient were on immunotherapy.

It's a real problem, because you have one word that patients may use meaning two very different things. It can get you into trouble if you are treating someone for potentially infectious causes rather than immunotherapyrelated adverse reactions, which are much more similar to graft-versus-host disease than in the case of traditional chemotherapy.

DR HENRY That's a very good point. I think, as we in oncology use these immunotherapies/checkpoint inhibitors more often, you will see them more often in the ED. Let's get right into that. You've identified this patient as not getting a traditional chemotherapy - hopefully, all our records on these patients are available. You've decided to follow onto what might be a side effect of the immunotherapy, so I'm going to name the side effects that always occur to me: lung, gastrointestinal (GI) - which could be loose bowels or liver function - rash, endocrine problems. Let's start with lung symptoms. You see the patient is short of breath and you identified immunotherapy. What's your next step?

DR SAMMON That's a great example, because the problem is that you see these patients with cough or shortness of breath and pulmonary complications, and pulmonary complications of immunotherapy, while rare, are potentially life threatening if they're not identified quickly.

You can start with a chest X-ray on these patients knowing, however, that for a good percentage of them you won't see findings on their chest X-ray (Figure 1, from Sammon M, Tobin T. Identification and management of immune-related adverse events in the emergency setting. Presented at: Advances in Cancer Immunotherapy - Society for Immunotherapy of Cancer (SITC); August 4, 2017; Philadelphia, PA). You need to proceed to computed tomography (CT), because the issue is that you can have protean findings on the $\mathrm{CT}$ related to immunotherapy treatment/adverse reactions. You need to have a very high index of suspicion regardless of what abnormal findings you're seeing on this $\mathrm{CT}$ and erring toward withholding the drugs, starting treatment, and being more aggressive with this type of finding.

DR HENRY I've heard you talk at a conference about a patient with metastatic lung cancer, or some other tumor that may have existing disease in lung. The patient is aware of that, and the chart reflects that. Then you have this difficulty where the $\mathrm{CT}$ scan shows a pneumonitis, and it may not be tumor progression at all - it may be the drug. How do you work through that? Of course, your additional problem is you don't have a whole lot of time. You must decide to whether you're going to keep them in the ED, admit them, or send them home.

DR SAMMON Right. One of the first things is to get the oncologist involved at an early stage in treating this. We are all a team. We are all working together, and it's very important to have that communication occur very early. I'm going to err on admitting those folks who have had symptoms and who have had findings on a chest $\mathrm{CT}$, because they can progress. They can get much worse. I'm going to be getting the oncologist involved very early. We're going to have the conversation about whether we should be starting steroids on them in the ED, getting them upstairs, and being aggressive in treating this.

DR HENRY So, time and therapy are very important.

\section{DR SAMMON Yes.}

DR HENRY You'll get that steroid started as an antidote right away.

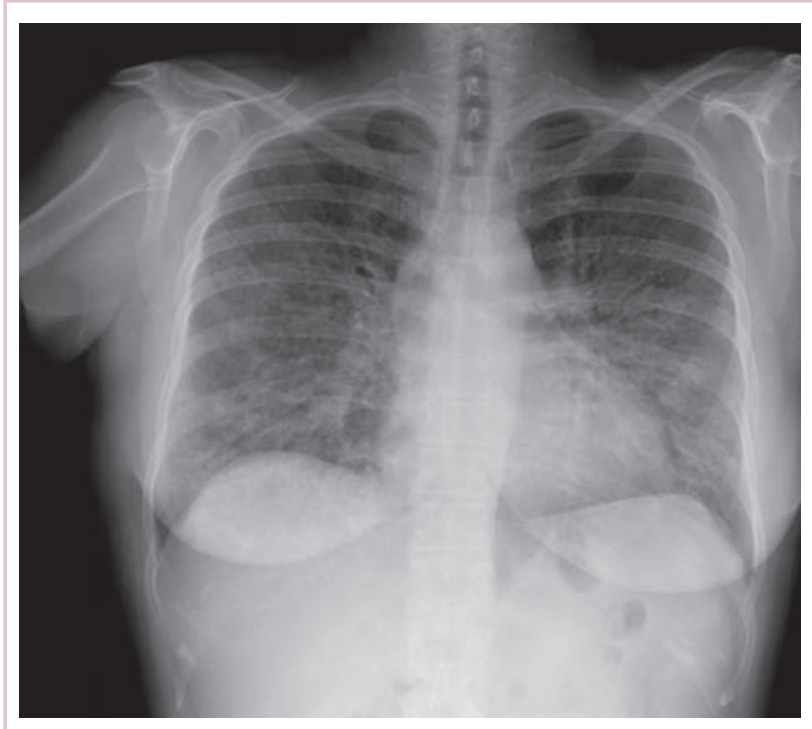

FIGURE 1 In patients with immunotherapy-related pulmonary complications, you can start with a chest X-ray, knowing that for a good percentage of them you won't see findings on the X-ray. Reproduced with permission from the Society for Immunotherapy of Cancer. 
DR SAMMON Absolutely. For grade 2, you're going to use methylprednisolone, $1 \mathrm{mg} / \mathrm{kg}$ daily. For anything higher than that - grade 3 or grade 4 - we're going to start higher. We're going to start at $2-4 \mathrm{mg} / \mathrm{kg}$ a day and get the patient upstairs and taper them slowly.

DR HENRY That's worth empathizing. I, sadly, had a patient who ultimately did well, but had severe grade 4 pneumonitis. The recommendation is $2-4 \mathrm{mg} / \mathrm{kg}$ per day of methylprednisolone, and he got through it and tapered slowly over many weeks. Of course, the patient would have long left your ER.

DR SAMMON Absolutely, but it's very important to work together as a team to make these patients have good outcomes.

DR HENRY Agree. Let's change over to the GI symptoms. The patient comes in and misidentifies him- or herself as having chemotherapy and diarrhea. We are used to causing nausea, vomiting, and diarrhea with some of our therapies. You realize, in talking to the oncologist, the patient is taking a checkpoint inhibitor. How would you approach the patient with diarrhea?

DR SAMMON First, I would talk to the patient. I would try to establish the baseline number of stools per day, because it's not defined as a definite number of stools per day, it's the number of stools above their baseline per day. If they're having fewer than 4 stools above their baseline per day, I would send off some tests. We can send off a C diff (Clostridium difficile) test, we can send off a stool culture - all the parasites. Make sure the oncologist is going to be able to get these results and get them followed up, because these are results I'm not going to get back myself in the ED.

Then I'm going to talk to the patient about symptomatic treatment. I'm going to talk to them about oral hydration, a bland diet. Avoid using loperamide or any other antidiarrheal medicines, because that could decrease the frequency of stools but mask more severe symptoms that they may be having.

If I have a patient who is having more than 4 stools above their daily baseline and it's been happening 4 to 6 stools a day for more than a week, I'm going to be sending those studies off, and I'm going to be having a conversation with the treating oncologist to find out if they want me to start the patient on steroids immediately, or if they want to wait for the test results to come back and have the steroids started as an outpatient.

These moderate patients can maybe wait a day until these test results come back. Those who are having more than 7 stools above baseline per day, peritoneal signs, ileus, or fever, are the patients you should worry about. You need to admit them for IV hydration. You need to do the stools, so you might need to keep them in the hospital until you find the results of the stool studies. You need to rule out perforation.

You may be starting steroids on these patients sooner rather than later. They're going to be getting systemic corticosteroids at about $1-2 \mathrm{mg} / \mathrm{kg}$ of prednisone equivalent, assuming there is no perforation and their stool studies are negative. If they are unstable, though, they are really going to need high-dose corticosteroids. They are going to need methylprednisolone, $125 \mathrm{mg} \mathrm{IV}$, to evaluate for their responsiveness. These folks really need to be treated as inpatients, and they need to have their oncologists involved early on with their treatment.

DR HENRY Yes. I couldn't agree with you more. When I talk to the diarrhea side effect patients that we see, I tend to think it's a curse. It's volume. It's calories. It's electrolytes. The number of stools you're mentioning, it is almost certainly going to need admission to rule out other causes. Then, if it's the checkpoint inhibitor, the steroid antidotes.

Let's move on to the rash. This is another organ system that can be affected by immunotherapy. What is your approach when you see a generalized body rash in a patient on one of these drugs who is sent to the ED?

DR SAMMON I am obviously going to be ruling out other causes first, but generally you're going to see a maculopapular rash. It may be itchy. It may be burning. Patients will often describe it as just sort of having a tight sensation. I'm going to be looking at them a little bit like I look at a burn patient. What is their total body surface area that's involved? If they've got less than $30 \%$ of their total body surface area involved, that's considered a mild reaction.

For those folks, I'm not going to use systemic steroids, but I can give them some topical steroids, and I can give them some Benadryl, some diphenhydramine, and really treat them symptomatically as well as ensuring that they have early follow-up to make sure this isn't progressing. Once we get between $30 \%$ and $50 \%$ body area, we're talking about a moderate toxicity. If these patients are not improving rapidly with just withholding the drug, they need systemic corticosteroids.

We usually treat them at about $0.5-1 \mathrm{mg} / \mathrm{kg}$ body weight a day of prednisone equivalent. Just as with burn patients, these patients' symptoms can become very severe. You can see signs of blistering, dermal ulceration, necrotic, bolus, hemorrhagic lesions (Figure 2, A-D). ${ }^{1}$ These folks can have very difficult-to-manage fluid balances, and they're at very high risk for skin infections as well. They need to be treated as inpatients. If possible, you might want to consider sending these patients to a burn unit. They need systemic corticosteroids, $1-2 \mathrm{mg} / \mathrm{kg}$ per day, and they need careful monitoring for signs of dehydration, electrolytic abnormalities, and/or skin infections. They need excellent wound care. 

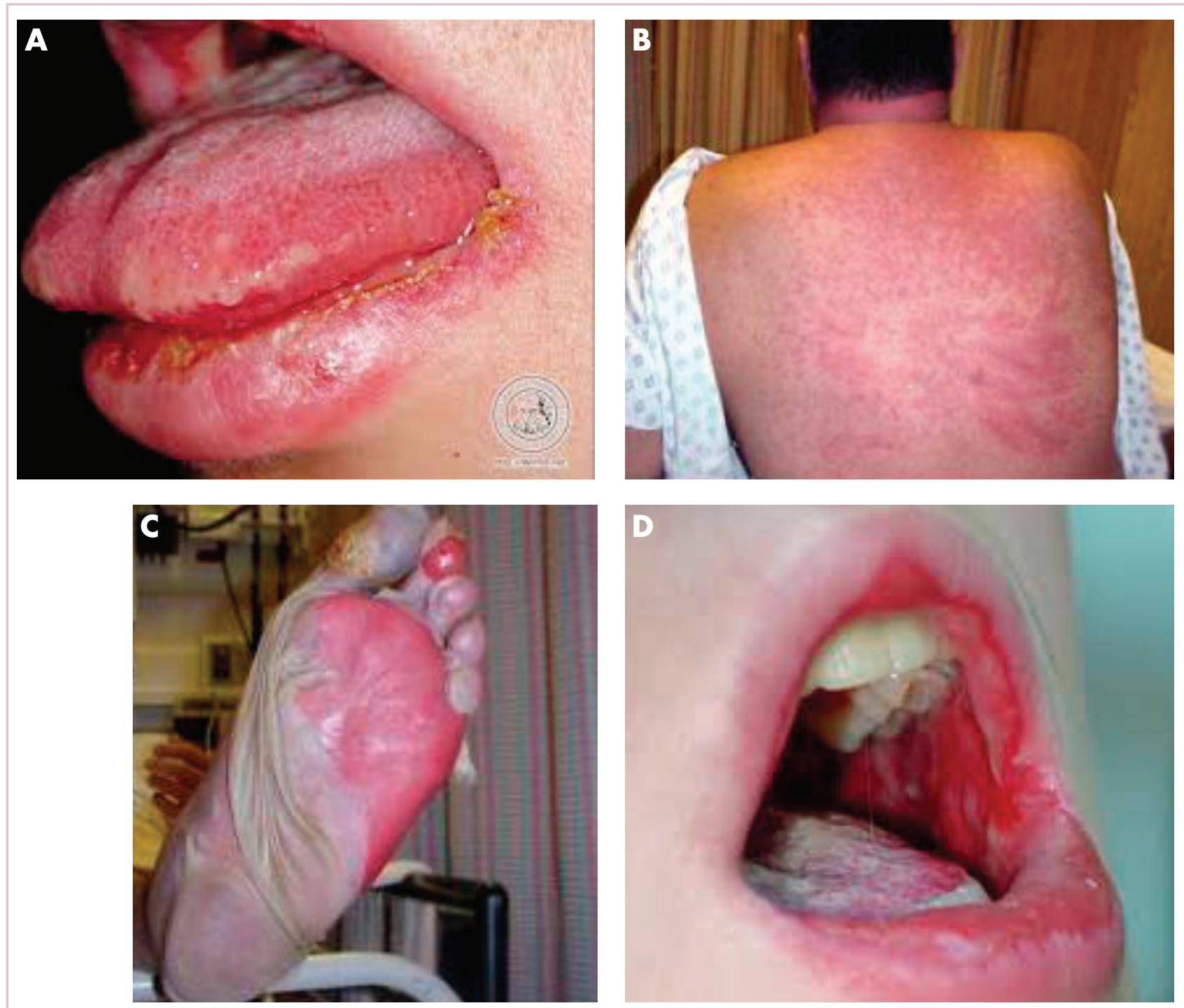

FIGURE 2 A patient with immunotherapy-related generalized body rash, showing A, blistering; B, dermal ulceration; C, necrotic bullous; D, hemorrhagic lesions. Reproduced with permission from the Society for Immunotherapy of Cancer.

DR HENRY That's very well put and always difficult, because there are so many causes of rash. That takes me to an area that has always been difficult for me, which is therapy-related endocrine problems. It's interesting to note that these drugs can cause endocrine problems. I've heard you speak about the pituitary affecting vision, as well as thyroid or adrenal issues. Let's start with how you'd approach vision difficulty in a patient on these drugs.

DR SAMMON The endocrinopathies that you can get with these checkpoint inhibitors really have a myriad of symptoms. Your patient may present saying that they're feeling tired, that they're feeling weak, or they may have a headache. If your patient is having actual pituitary enlargement, they can present with headaches, visual field defects, or cranial nerve defects. The reason for those symptoms is that the pituitary sits in the cavernous sinus, and you have various cranial nerves passing through that area as well as the optic chiasm just above the pituitary gland (Figure 3). ${ }^{1}$ Your patient may present with a bitemporal hemianopia. Or with diplopia. You are going to want to very quickly get either a CT scan or an MRI to find out if that is what's going on (Figure 4). ${ }^{1}$ These folks need to be treated aggressively as well.

DR HENRY You'll get your CT scan or your MRI and rule out an enlargement or a change to the visual field. I haven't seen this yet, but certainly exciting when you see it to treat it. Would you get the radialis brevis involved, steroids involved? How would you manage that? 


\section{Feature}

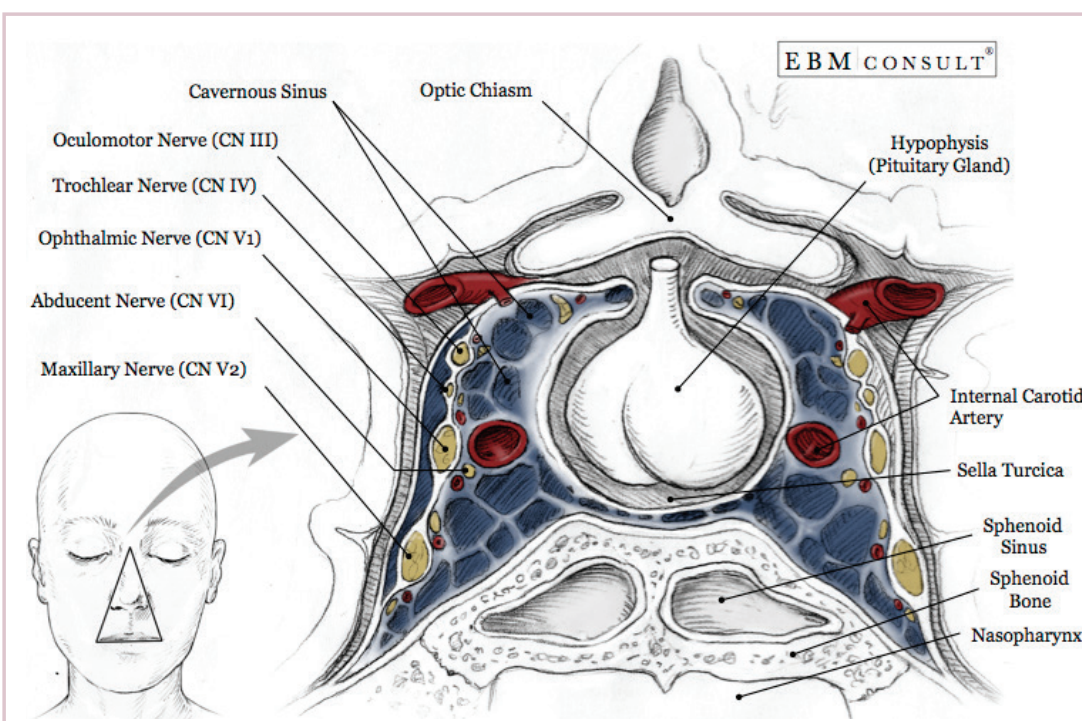

FIGURE 3 The pituitary gland sits in the cavernous sinus, and various cranial nerves pass through this area as well as the optic chiasm just above the gland. Reproduced with permission from the Society for Immunotherapy of Cancer.
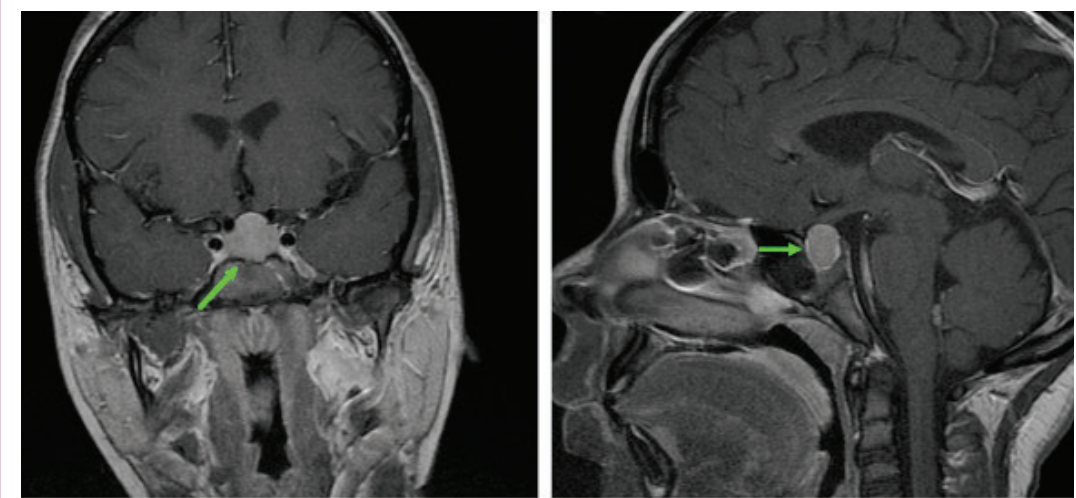

FIGURE 4 For patients presenting with immunotherapy-related bitemporal hemianopia or diplopia, you need to very quickly get a CT or an MRI to confirm if that is what's going on. Reproduced with permission from the Society for Immunotherapy of Cancer. you may want to choose using dexamethasone, because you can still do the corticotropin stimulation test. However, if your patient is in frank shock because of what you think is an adrenal crisis, you're going to want to use hydrocortisone. If a patient is truly hypotensive and unstable, the testing is at that point less important than the treatment.

DR HENRY Very interesting. We have covered what I would consider the major aspects of these fascinating drugs. We haven't covered all of what they do when they work well, which hopefully we're seeing more and more often, but we have covered very well what can happen when things go wrong in side effects. Anything else that you would like to add from the ED perspective or other side effects worth mentioning?

DR SAMMON The thing that I would most like to share with the oncology office is the importance of communicating with your patients that, when they're on these drugs, they need to tell emergency physicians that they're on immunotherapy, not chemotherapy. It might be helpful to give these patients a card stating that they're on immunotherapy, not chemotherapy, and outlining some of the side effects that ED physicians should be looking out for in these patients.

DR HENRY That's a great point. I've seen that some of the manufacturers have little cheat cards that the patient can carry naming the drug and the side effects, because not all ED doctors are aware of the side effects of these drugs.

DR SAMMONAbsolutely. We love those cards.
DR SAMMON It's interesting, because you do want to use corticosteroids. One of the questions here is, which corticosteroid do you want to use? If you're talking about someone who may have adrenal insufficiency, you may want to be able to do a stimulation test. In these patients,
DR HENRY Yes. I've also given some to the ED doctors at Pennsylvania Hospital, and they love it. I think we've covered everything in quite a bit of detail. Thank you, Dr Sammon, for sharing this information from the frontlines of the ED. 